PAPER

\section{Exciton induced directed motion of unconstrained atoms in an ultracold gas}

To cite this article: K Leonhardt et al 2017 J. Phys. B: At. Mol. Opt. Phys. 50054001

View the article online for updates and enhancements.

\section{Related content}

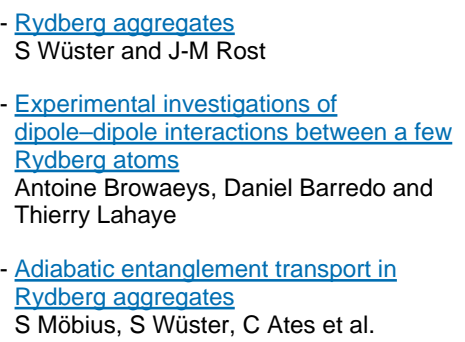

\section{Recent citations}

\author{
Rydberg aggregates \\ S Wüster and J-M Rost \\ - Quantum dynamics of long-range \\ interacting systems using the positive- $\mathrm{P}$ \\ and qauge- $P$ representations \\ S. Wüster et al
}

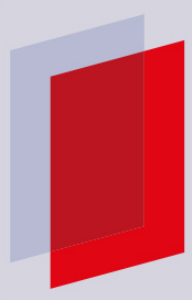

\section{IOP ebooks'}

Bringing you innovative digital publishing with leading voices to create your essential collection of books in STEM research. Start exploring the collection - download the first chapter of every title for free. 


\title{
Exciton induced directed motion of unconstrained atoms in an ultracold gas
}

\author{
K Leonhardt ${ }^{1}$, S Wüster ${ }^{1,2,3}$ and J M Rost ${ }^{1}$ \\ ${ }^{1}$ Max Planck Institute for the Physics of Complex Systems, Nöthnitzer Strasse 38, D-01187 Dresden, \\ Germany \\ ${ }^{2}$ Department of Physics, Bilkent University, 06800 Çankaya, Ankara, Turkey \\ ${ }^{3}$ Department of Physics, Indian Institute of Science Education and Research, Bhopal, Madhya Pradesh 462 \\ 023, India
}

E-mail: karlo@pks.mpg.de

Received 3 August 2016, revised 21 December 2016

Accepted for publication 4 January 2017

Published 14 February 2017

\begin{abstract}
We demonstrate that through localised Rydberg excitation in a three-dimensional cold atom cloud atomic motion can be rendered directed and nearly confined to a plane, without spatial constraints for the motion of individual atoms. This enables creation and observation of nonadiabatic electronic Rydberg dynamics in atoms accelerated by dipole-dipole interactions under natural conditions. Using the full $l=0,1 m=0, \pm 1$ angular momentum state space, our simulations show that conical intersection crossings are clearly evident, both in atomic position information and excited state spectra of the Rydberg system. Hence, flexible Rydberg aggregates suggest themselves for probing quantum chemical effects in experiments on length scales much inflated as compared to a standard molecular situation.
\end{abstract}

Keywords: excitons, Rydberg atoms, ultra cold gases

(Some figures may appear in colour only in the online journal)

\section{Introduction}

Electronic Rydberg excitation in ultracold gases creates highly controllable quantum systems with promising applications that take advantage of the extreme interactions among Rydberg atoms $[1,2]$. Prominent examples include quantum information [3-6], the simulation of spin systems [7-9] and many more processes with controlled electron correlation. Typically, for these applications the atomic gas is assumed to be 'frozen'. The unavoidable (thermal) motion of the atoms constitutes then a limiting source of noise and decoherence $[10,11]$.

Yet we know, that in every molecule bound atomic and electronic motion are entangled in coherent dynamics. Analogously, atoms of an ultra cold gas-energetically in the continuum - can be turned from a noise source into an asset using Rydberg aggregates [12-18]. Rydberg excitation realised as an exciton that entangles two or more atoms exerts a well-defined mechanical force on the atoms which start to move. The resulting directed motion of a few Rydberg atoms $[16,17,19-27]$ enables transport of electronic coherence along with atomic mechanical momentum involving quintessential quantum chemical processes such as conical intersections (CI) [18, 20, 27, 28]. Thereby, transport of energy and entanglement could be ported from the (chemical) $\mathrm{nm}$ scale to spatial distances of $\mu \mathrm{m}[17,19,27,29]$, allowing for direct and detailed optical monitoring [15, 30-33] of quantum many-body state dynamics. To distinguish the continuum motion of the atoms from the usual bound (vibrational) motion in standard aggregates we call our systems flexible Rydberg aggregates. However, the prerequisite of this directed continuum motion in $[17,19,27]$ was an external confinement of the atoms to one-dimensional chains. While eventually possible in tight atom traps of experiments in the future, this dimensionally reduced environment is not only a complication for the experiment but also a principal restriction in our quest to take chemical coherence of atoms bound in molecules to atoms moving in the continuum.

In the following, we will show how to lift this restriction by demonstrating that if the Rydberg atoms are prepared in a low dimensional space, e.g., a plane, the ensuing entangled molecular motion in the continuum will remain confined to 


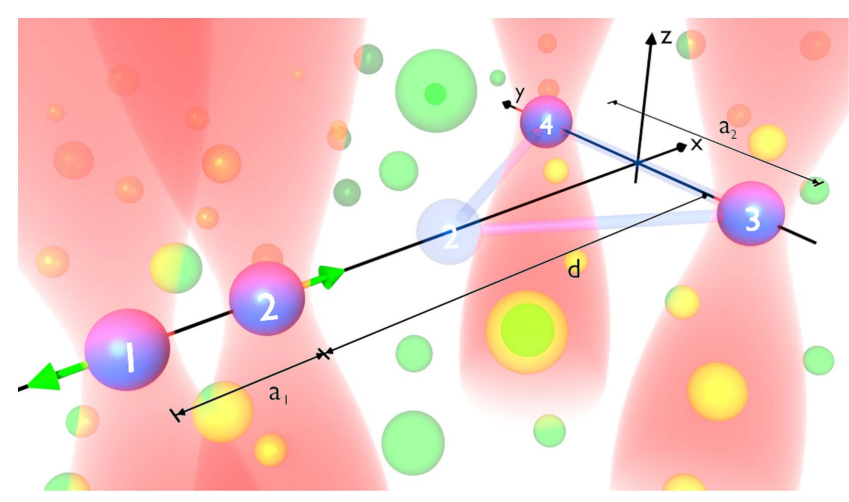

Figure 1. Embedded Rydberg aggregate. Four excitation beams (red shades) define focus volumes in which exactly one atom is excited to a Rydberg state (blue balls, 1-4), within a cold gas (green balls). Our co-ordinate system has its origin at the mean position of atom 1 , several geometrical parameters are explained in the text. Subsequent to Rydberg excitation, dipole-dipole interactions will cause acceleration along the green arrows, causing atom 2 to reach the position shown in light blue, where a CI will cause strong non-adiabatic effects.

this space despite the possibility for all particles of the flexible aggregate (ions and electrons) to move in full space. Together with advances in the newest generation experiments on Rydberg gases beyond the frozen gas regime, involving microwave spectroscopy [34] or position sensitive field ionisation [35, 36], our results enable the quantum simulation of nuclear dynamics in molecules using Rydberg aggregates as an experimental science. These recent efforts [34-36] extend earlier pioneering studies of motional dynamics in Rydberg gases [37-45] and now render the rich dynamics of Rydberg aggregates fully observable. Complementary ideas suggesting the quantum simulation of electronic dynamics in molecules with cold atoms as can be found in [46].

\section{Preparation of the Rydberg system}

\subsection{Localised excitation of single Rydberg atoms by laser light in an ultracold gas}

A central element of the Rydberg aggregate, non-adiabatic motional dynamics on several coupled Born-Oppenheimer (BO) surfaces [20, 27], is now experimentally accessible, as we show here. To be specific, we investigate a Rydberg aggregate consisting of $N=4$ Rydberg ${ }^{7} \mathrm{Li}$ atoms (mass $M=11000 \mathrm{au}$ ), excited to the principal quantum number $\nu=80$, embedded within a cloud of cold ground-state atoms, see figure 1. This setup is created by Rydberg excitation of single atoms in the gas with tightly focused lasers. We assume that the focus volumes are small enough to deterministically excite just a single atom within each focus to a Rydberg $s$-state (angular momentum $l=0$ ), exploiting the dipoleblockade [3, 4].

As shown in figure 1, the Rydberg atoms attain a T-shaped configuration after excitation, defined by laser foci centred on the mean positions $\mathbf{R}_{0}^{(\alpha)}$ for atoms $\alpha=1, \ldots, N$.
We place the origin of our coordinate system at $\mathbf{R}_{0}^{(1)}$, and define the directions $\mathbf{e}_{x}:=\mathbf{R}_{21} /\left\|\mathbf{R}_{21}\right\|, \mathbf{e}_{y}:=\mathbf{R}_{43} /\left\|\mathbf{R}_{43}\right\|$, where $\mathbf{R}_{\alpha \beta} \equiv \mathbf{R}_{0}^{(\alpha)}-\mathbf{R}_{0}^{(\beta)}$ denotes the mean interatomic distance vectors. The mean positions of the atoms are then given in the cartesian basis $\left\{\mathbf{e}_{x}, \mathbf{e}_{y}, \mathbf{e}_{z}\right\}$, shown in the figure, by $\mathbf{R}_{0}^{(2)}=$ $\left(a_{1}, 0,0\right), \quad \mathbf{R}_{0}^{(3)}=\left(a_{1}+d,-a_{2}, 0\right) \quad$ and $\quad \mathbf{R}_{0}^{(4)}=\left(a_{1}+d\right.$, $\left.a_{2}, 0\right)$. The geometrical parameters employed here are $a_{1}=10 \mu \mathrm{m}, a_{2}=37 \mu \mathrm{m}$ and $d=51 \mu \mathrm{m}$. Importantly, the initial spatial configuration of the aggregate spans a plane in $3 \mathrm{D}$ space. We will refer to atoms $(1,2)$ as the $x$-dimer and atoms $(3,4)$ as the $y$-dimer. The positions of all Rydberg atoms are collected into the vector $\mathbf{R} \equiv\left(\mathbf{R}^{(1)}, \ldots, \mathbf{R}^{(N)}\right)^{\mathrm{T}}$. Co-ordinates of ground-state atoms are not required since these will be merely spectators for the dynamics of Rydberg atoms, as shown in [23] and found experimentally in [34-36].

To be specific we assume a gas density of $\rho \approx 1 \times 10^{12}$ $\mathrm{cm}^{-3}$ and a temperature of $T=1 \mu \mathrm{K}$. For these parameters, Maxwell-Boltzmann statistics is applicable with velocities of the atoms normally distributed in each direction with variance $\sigma_{v}^{2}=k_{\mathrm{B}} T / M$, where $k_{\mathrm{B}}$ denotes the Boltzmann constant. The positions of atoms are randomly distributed in the foci of the excitation lasers, approximately given by the waist size $\sigma_{0}$ of the Gaussian beam. We assume the resulting probability distribution for the positions of Rydberg atoms after excitation to be

$$
\rho(\mathbf{R}, t=0)=\left(\pi \sigma_{0}^{2}\right)^{-3 N / 2} \mathrm{e}^{-\left|\mathbf{R}-\mathbf{R}_{0}\right|^{2} / \sigma_{0}^{2}},
$$

with $\sigma_{0}=0.5 \mu \mathrm{m}$. This isotropic spatial distribution differs from those in our previous studies [18, 27], where uncertainties where considered only in specific directions.

After laser excitation, the aggregate is in the electronic state $|S\rangle \equiv|s \ldots s\rangle$.

\subsection{Creating a p-excitation}

Following the laser-excitation, a microwave pulse, linearly polarised in a direction $\mathbf{q}$ which also serves as quantisation axis, transfers the Rydberg atoms from $|S\rangle$ to a repulsive exciton state $\left|\varphi_{\text {ini }}\right\rangle$ in which a single $(p, m)$-excitation (angular momentum $l=1$ ) with magnetic quantum number $m=0$ is coherently shared between atoms $(1,2)$, while atoms $(3,4)$ remain in the Rydberg $s$-state, $\left|\varphi_{\text {ini }}\right\rangle \approx$ $(|s(p, 0)\rangle+|(p, 0) s\rangle) / \sqrt{2} \otimes|s s\rangle$. See appendix $\mathrm{C}$ for further details on the microwave excitation. Selective excitation of this exciton is achieved by detuning the microwave frequency by the initial exciton energy $U_{\text {ini }}\left(\mathbf{R}_{0}\right) \approx 22.27 \mathrm{MHz}$ from the $s \rightarrow p$ transition. This energy shift addresses the second most energetic BO-surface, see appendix A for the definition of the BO-surfaces. Note that detuning the microwave by $U_{\text {ini }}\left(\mathbf{R}_{0}\right)$ ensures the creation of just a single $p$-excitation since all states with more $p$-excitations are off-resonant.

The full initial state is given by the density matrix

$$
\hat{\varrho}(t=0)=\hat{\varrho}_{0}^{\text {nuc }} \otimes\left|\varphi_{\text {ini }}\right\rangle\left\langle\varphi_{\text {ini }}\right|,
$$

where $\left\langle\mathbf{R}\left|\varrho_{0}^{\text {nuc }}\right| \mathbf{R}\right\rangle$ is the initial probability distribution $\rho(\mathbf{R}, t=0)$ given in (1). 
The initial state preparation sequence described so far would be similarly required in our other proposals regarding flexible Rydberg aggregates as discussed in [19]. However, only in this article do we allow for entirely unconstrained atomic motion in three-dimensions, all Rydberg atom angular momentum states $l=0,1 ; m=0, \pm 1$ and the anisotropy of dipole-dipole interactions which we will discuss nextly.

\section{Full dimensional dynamics with anisotropic dipole-dipole interactions}

The $p$-excited atom introduces resonant dipole-dipole interactions into the system. We expand the electronic wavefunction in the discrete basis $\mathcal{B}=\left\{\left|\pi_{\alpha}, m\right\rangle\right\}_{m=-1,0,1}^{\alpha=1, \ldots, N}$, where $\left|\pi_{\alpha}, m\right\rangle \equiv|s \ldots(p, m) \ldots s\rangle$ denotes the $N$-Rydberg-atom state with the $\alpha$ th atom in a $p$-state with magnetic quantum number $m$, while the other $N-1$ atoms are in an $s$-state. We thus neglect spin-orbit coupling, which is a good approximation for Lithium [18, 47].

Our effective electronic Hamiltonian model captures the essential features of atomic interactions

$$
\hat{H}_{\mathrm{el}}(\mathbf{R}):=\hat{H}_{\mathrm{dd}}(\mathbf{R})+\hat{H}_{\mathrm{vdW}}(\mathbf{R}),
$$

with $\hat{H}_{\mathrm{dd}}(\mathbf{R})$ containing the resonant dipole-dipole interactions between two atoms in different states $(s p)$ and $\hat{H}_{\mathrm{vdW}}(\mathbf{R})$ containing the non-resonant van-der-Waals (vdW)-interactions between two atoms in the same state ( $s s$ or $p p$ ). The resonant contribution is given by

$$
\hat{H}_{\mathrm{dd}}(\mathbf{R}):=\sum_{\substack{\alpha, \beta=1 ; \\ \alpha \neq \beta}}^{N} \sum_{\substack{m, m^{\prime}=-1 \\ 1}} V_{m, m^{\prime}}\left(\mathbf{R}_{\alpha \beta}\right)\left|\pi_{\alpha}, m\right\rangle\left\langle\pi_{\beta}, m^{\prime}\right|,
$$

with the dipole-dipole transition matrix element [48]

$$
\begin{aligned}
V_{m, m^{\prime}}(\mathbf{r}):= & -\sqrt{\frac{8 \pi}{3}} \frac{\mathfrak{d}^{2}}{\|\mathbf{r}\|^{3}}(-1)^{m^{\prime}}\left(\begin{array}{ccc}
1 & 1 & 2 \\
m & -m^{\prime} & m^{\prime}-m
\end{array}\right) \\
& \times Y_{2, m^{\prime}-m}\left(\theta_{\mathcal{Q}}, \phi_{\mathcal{Q}}\right),
\end{aligned}
$$

where $Y_{l m}$ are spherical harmonics and the six numbers enclosed by round brackets specify the Wigner- $3 \mathrm{j}$ symbol. We denote the radial matrix element with $\mathfrak{d}=\mathfrak{d}_{\nu, 1 ; \nu, 0}$ for a transition $\nu, s \rightarrow \nu, p$, such that $\mathfrak{d}=8250$ au for $\nu=80$. The angles

$$
\begin{gathered}
\theta_{\mathcal{Q}}:=\arccos \frac{\langle\mathbf{q}, \mathbf{r}\rangle}{\|\mathbf{r}\|}, \\
\phi_{\mathcal{Q}}:=\operatorname{atan} 2\left(\left\langle\mathbf{q}_{y}, \mathbf{r}\right\rangle,\left\langle\mathbf{q}_{x}, \mathbf{r}\right\rangle\right)
\end{gathered}
$$

are the polar and azimuthal angle of the interatomic distance vector $\mathbf{r}$ represented in the rotated orthonormal basis $\mathcal{Q}=\left\{\mathbf{q}_{x}, \mathbf{q}_{y}, \mathbf{q}\right\}^{4}$. This representation fixes the microwave polarisation direction $\mathbf{q}$ as quantisation axis. We will now first focus on the choice $\mathbf{q}=\mathbf{e}_{y}$ for our quantisation axis, and later also explore $\mathbf{q}=\mathbf{e}_{z}$ in section 6 .

\footnotetext{
4 The vectors $\mathbf{q}_{x}, \mathbf{q}_{y}$ are chosen to complete a right-handed cartesian coordinate system. They can be constructed by setting $\mathbf{q}_{x}:=\frac{\mathbf{a} \times \mathbf{q}}{\sqrt{\|\mathbf{a}\|^{2}-\langle\mathbf{a}, \mathbf{q}\rangle^{2}}}$ and $\mathbf{q}_{y}:=\mathbf{q} \times \mathbf{q}_{x}$, in which $\mathbf{a} \nVdash \mathbf{q}$ can be chosen arbitrarily, not changing the physics.
}

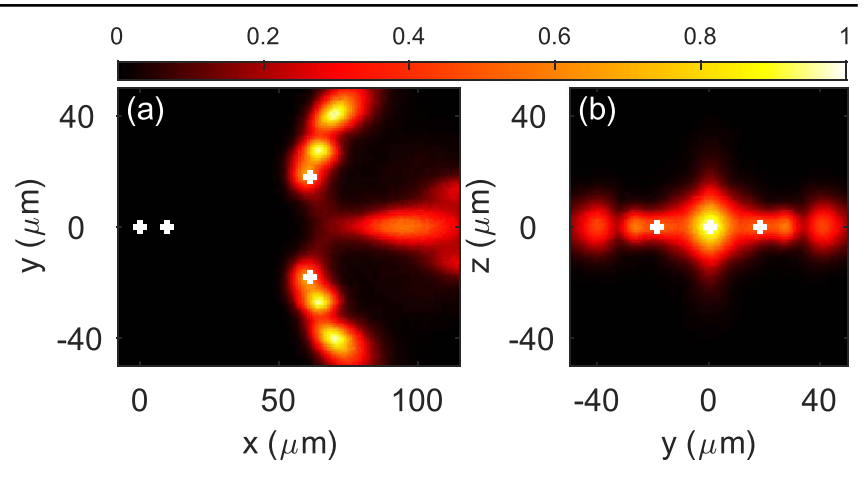

Figure 2. Atomic density $n(\mathbf{r}, t)$ (see appendix D) of the final state at $t=92.9 \mu \mathrm{s}$, using $\mathbf{q}=\mathbf{e}_{y}$. Shown are column densities, (a) in the $x-y$ plane, $\bar{n}(x, y, t)$, and (b) in the $y-z$ plane, $\bar{n}(y, z, t)$, see appendix D. The white ' + ' mark the initial atomic positions. The maximal densities are set to 1 .

The non-resonant vdW-Hamiltonian $\hat{H}_{\mathrm{vdW}}(\mathbf{R}):=$ $-\mathbb{1} \sum_{\alpha, \beta=1 ; \alpha \neq \beta}^{N} C_{6} /\left(2\left|\mathbf{R}_{\alpha \beta}\right|^{6}\right)$, assumes identical interactions for $s$ - and $p$-states for simplicity. In reality they typically differ, resulting in interesting effects at shorter distances [22] that will not be relevant here.

Since no additional magnetic field is present in (3), all quantum numbers $m$ will contribute to the dynamics.

\section{Motion of the Rydberg atoms: non-adiabatic dynamics}

We are now in a position to follow the motion of the Rydberg atoms, which sets in as a consequence of the exciton formation. Motion and exciton dynamics are modelled with a quantum-classical approach, described in appendixes A and B. The four Rydberg atoms of the aggregate will move essentially unperturbed through the background gas [23]. This motion takes place in three-dimensional space and is governed by anisotropic resonant dipole-dipole interactions without any confinement. Initially atoms 1 and 2 repel each other as sketched in figure 1 . Eventually atom 2 comes closer to atoms 3 and 4 setting them into motion as well. The motion remains confined near the $x-y$-plane, facilitating observations. The total atomic column densities (the total density in our case is just the sum over the spatial probability distributions of each atom, see appendix D) after some time of free atomic motion have an interesting multi-lobed structure, shown in figure 2, a central result of the present work. With the mechanical momentum transfer in mind, one would expect that atom 1 moves to the left in figure 2(a) and atom 2 to the right. Both is indeed the case (at $93 \mu$ s atom 1 is no longer within the range of the figure). However, atom 2 has an elongated density profile along the $x$-axis at the final time. Moreover, one would expect the two atoms 3 and 4 to move outwards on the $y$-axis after the kick by atom 2 . Although this is the case, the densities reveal two positions for each of them. The reason for this behaviour is that the electronic population gets distributed over two states (BO-surfaces) by traversing a $\mathrm{CI}$ at about $20 \mu \mathrm{s}$. The CI occurs when atoms 2-4 nearly form an equilateral triangle. As a consequence, two BO-surfaces 


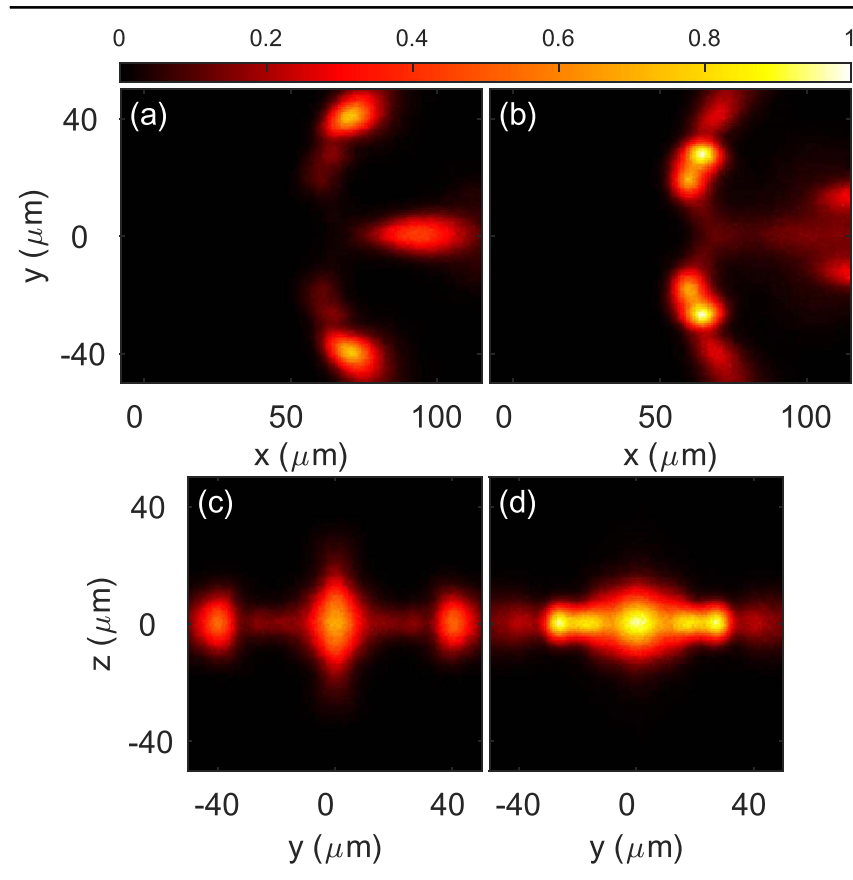

Figure 3. As in figure 2, but with atomic density segregated according to BO surfaces: (a) and (c) second highest BO surface, (b) and (d) fourth highest BO surface. (a) and (b) column densities in the $x-y$-plane. (c) and (d) column densities in the $y$-z-plane. The global maximum for each column density is set to 1 .

are populated almost equally and exert different forces on the atoms. This explains the 'double'-appearance of the final positions for atoms 3 and 4 and the blurred final position for atom 2. Figure 2(b) demonstrates that the entire dynamics indeed remains confined near the $x-y$-plane, which also facilitates the interpretation in terms of the BO-surfaces. Figure 3 shows atomic densities segregated according to the two involved BO-surfaces and confirms the interpretation just given.

Note, that motion proceeds on the lower of the two surfaces when the CI was missed due to a configuration asymmetric in $y$. This causes one of the $y$-dimer atoms to stay mostly at its initial position (maxima at $x=61 \mu \mathrm{m}$, $y= \pm 18.5 \mu \mathrm{m}$ ), while the second receives a kick. In the density profiles this results in a total of four maxima for this surface, corresponding to kick and no-kick for both atoms 3 and 4. We discussed this phenomenology in more detail earlier [18, 27].

The atomic motion in two orthogonal directions causes transfer of $p$-excitation from the initial $m=0$ orientation to the $m= \pm 1$ orientations, as can be seen in figure 4 . The figure shows the spatial distribution of the $p$-excitation segregated by magnetic quantum number, $\rho_{\text {exc,m }}(\mathbf{r}, t)$, see appendix D. This is caused by the anisotropy of the dipoledipole interactions, which is not present for one-dimensional geometries of the aggregate [17, 19].

Optical confinement of Rydberg atoms in one-dimensional traps along with a reduction of the electronic state space assumed in our related earlier work [20, 27] constitute a significant experimental challenge. The present results show that these restrictions are not required. It is simply the

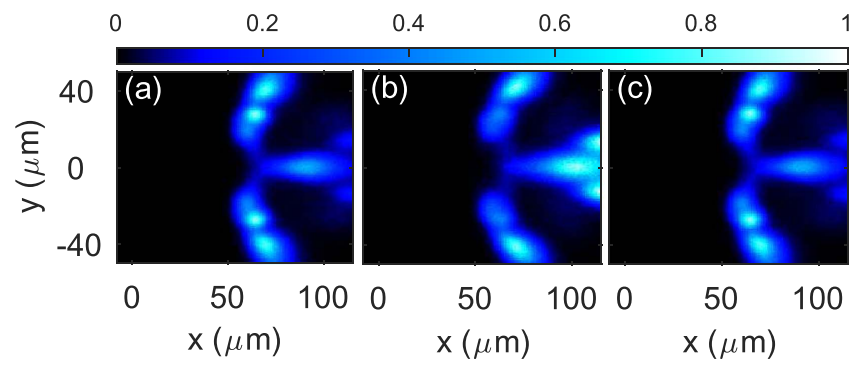

Figure 4. Spatial distribution of the $(p, m)$-excitation at $t=92.9 \mu \mathrm{s}$. Shown is the $x-y$ column density, segregated by magnetic quantum numbers: (a) $m=-1, \rho_{\mathrm{exc},-1}(x, y, t)$, (b) $m=0, \rho_{\mathrm{exc}, 0}(x, y, t)$ and (c) $m=1, \rho_{\mathrm{exc}, 1}(x, y, t)$, see also appendix $\mathrm{D}$. The global maximal density of excitation over all $m$-levels is set to 1 .

symmetry of the initially prepared system which keeps the motion similarly planar and hence accessible. The successful splitting into different motional modes through the CI is a sensitive measure for the extent to which the atomic motion remains in a plane. Our findings suggest Rydberg aggregates as an experimental platform for the study of quantum chemical effects on much-inflated length and time scales with presently available technologies [15, 34-36, 49].

\section{Considerations for an experiment}

\subsection{Experimental signatures}

The total atomic density of figure 2 is experimentally accessible if the focus positions $\mathbf{R}_{0}^{(n)}$ are sufficiently reproducible to allow averaging over many realisations. Additionally, one requires near single-atom sensitive position detection. A shotto-shot position uncertainty $\sigma_{0}$ in 3D within each laser focus is already taken into account in our simulation. Recent advances in position sensitive field ionisation enable $\sim 1 \mu \mathrm{m}$ resolution, clearly sufficient for an image such as figure 2 . The data for panel (b) could alternatively also be retrieved by waiting for atoms 3 and 4 to impact on a solid state detector. The background gas can also act as a probe for position and state of the embedded moving Rydberg atoms [15, 30-33], offering resolution sufficient for figure 2 .

Non-adiabatic dynamics discussed here can not only be monitored in position space, but also in the excitation spectrum of the system, similar to [34]. To obtain the timeresolved potential energy density $u(E, t)$, we bin the potential energy $U_{s}(t)$ of the currently propagated BO-surface $s$ (see appendix B) into a discretized energy grid $E$ and average over all trajectories. Thereby, one can elegantly visualise electron dynamics on two BO-surfaces subsequent to CI crossing, as can be seen in figure $5(\mathrm{a})$.

Observation of $u(E, t)$ could proceed by monitoring the time- and frequency-resolved outcome of driving the $p \rightarrow d$ transition. Similar techniques would allow an observation of the entire exciton spectrum of the system (with possibly unoccupied states), rather than only the currently populated state. The corresponding exciton density of states $g(E, t)$ analogous to $u(E, t)$, but now with all eigenenergies $U_{k}$ 


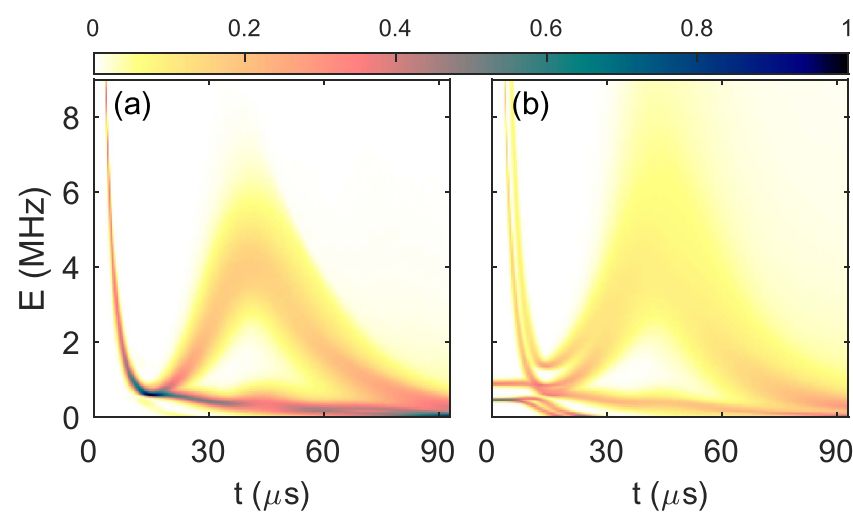

Figure 5. Time-resolved spectra of (a) potential energy $u(E, t)$ (as explained in the text) and (b) exciton density $g(E, t)$ of all states. The maximum has been set to one for each density. To emphasise low density features, we plot $\sqrt{u(E, t)}$ and $\sqrt{g(E, t)}$, respectively. Such spectra are experimentally observable through time-resolved microwave spectroscopy [34].

binned instead of only the currently propagated surface $U_{s}$, is shown in figure $5(\mathrm{~b})$.

\subsection{Limits on laser waists and temperatures}

Observable splitting of the dynamics onto different BO-surfaces critically relies on guiding the atomic configuration close to a CI location with the right spatial widths and velocities. The waist of the laser focus primarily determines the position uncertainty of the initial spatial configuration of the aggregate, which impacts the population ratio of the relevant BO surfaces. For larger waists, the distinct density features in the atomic density become blurred, such that it is no longer possible to clearly assign parts of the atomic density to dynamics along a particular BO surface. This is already the case for a waist size of $\sigma_{0}=1 \mu \mathrm{m}$, as apparent from figure 6(b). An additional blurring is due to the temperature of the gas, through the velocity distribution.

However, the density profiles are more sensitive to changes in focus size than to temperature. This is verified in figure 6: a doubling of the temperature from $T=1 \mu \mathrm{K}$ (first row in figure 6) to $T=2 \mu \mathrm{K}$ (second row in figure 6) still allows an identification of multi-BO features in the atomic densities for a laser waist size of $\sigma_{0}=0.5 \mu \mathrm{m}$, as apparent in figure 6(a). Only for temperatures around $T=4 \mu \mathrm{K}$ can the multiple density features no longer be identified. Nevertheless, even in this case, the CI still leaves its mark in the potential energy spectrum in the form of a distinct branching. This is even the case for a temperature of $T=4 \mu \mathrm{K}$ and a laser waist size of $\sigma_{0}=1 \mu \mathrm{m}$, as can be seen in figure 7 .

\subsection{Perturbation by ground state atoms}

We expect the dynamics of the embedded Rydberg aggregate discussed here not to be significantly perturbed by its cold gas environment. Rydberg-Rydberg interactions substantially exceed elastic Rydberg ground-state atom interactions [50, 51] for separations $d>200 \mathrm{~nm}$, and dipole-dipole excitation transport disregards ground state atoms [23]. Our kinetic energies of $\mathcal{O}(10 \mathrm{MHz})$, from dipole-dipole induced motion, are still low enough to render inelastic $\nu$ or $l$ changing collisions very unlikely [51], leaving molecular ion- or ion pair creation as main Rydberg excitation loss channel arising from collisions with ground state atoms [51, 52]. Thermal motion is even slower. When considering loss channels while assuming a background gas density of $\rho=1 \times 10^{12} \mathrm{~cm}^{-3}$, we can extrapolate experimental data from $\mathrm{Rb}$ [34] to infer a lifetime of about $\tau=530 \mu \mathrm{s}$ for the embedded Rydberg aggregate (see appendix E). However, detrimentally large cross sections for the same processes were found in [51, 52] for much larger densities $\rho$. Further research on ionisation of fast Rydberg atoms within ultracold gases is thus of interest for the present proposal.

\subsection{Alternative initialisation of the aggregate: trapping, cooling and excitation of atoms in optical tweezers}

An alternative to initialise the Rydberg aggregate through direct excitation of atoms in the gas would be to individually trap four atoms in optical tweezers [53] at positions $\mathbf{R}_{0}^{(n)}$, with trapping width $\sigma_{0}$, prior to Rydberg excitation, see e.g. [49]. Single atoms can be cooled to the vibrational ground state of optical tweezers [54], after which the atomic wave function approximately realises the ground state of a harmonic oscillator. The initial position uncertainty of the aggregate then is determined by the trapping frequencies $\omega$ of the optical tweezers. An uncertainty of $\sigma_{0}=0.5 \mu \mathrm{m}$ for the location of each atom, as used for the results in section 4 , requires a trapping frequency, $\omega / 2 \pi=\hbar /\left(2 \pi M \sigma_{0}^{2}\right)$, of about $1 \mathrm{kHz}$, which is experimentally achievable [54]. A population of the vibrational ground state above $99 \%$ is reached for temperatures below $70 \mathrm{nK}$ for this trapping frequency. This ground state yields a variance for the velocity, $\sigma_{v}^{2}=\hbar \omega / 2 M$, of only $17 \%$ of the value corresponding to the ideal gas at $T=1 \mu \mathrm{K}$, as used in section 4 .

\section{Switching of BO surfaces}

The present system allows a simple handle deciding on which BO surface the system is initialised, and consequently to what extent the subsequent evolution involves CIs and non-adiabatic effects. This handle is the linear polarisation direction of the microwave for exciton creation, which selects the exciton state that is initially excited.

So far we have discussed the case $\mathbf{q}=\mathbf{e}_{y}$. Choosing $\mathbf{q}=\mathbf{e}_{z}$ instead allows the same $s \rightarrow p$ excitation pulse to access a different initial BO-surface, with substantially less non-adiabaticity. This is shown in figure 8 . The dramatic difference to the corresponding earlier results in figures 2 and 5 can be viewed as a dependence on the magnetic quantum number of our initial state. With the microwave polarisation direction along $\mathbf{e}_{z}$, the third most energetic exciton is excited, which would contain $m= \pm 1$ population using the previously chosen excitation direction. 
(a)

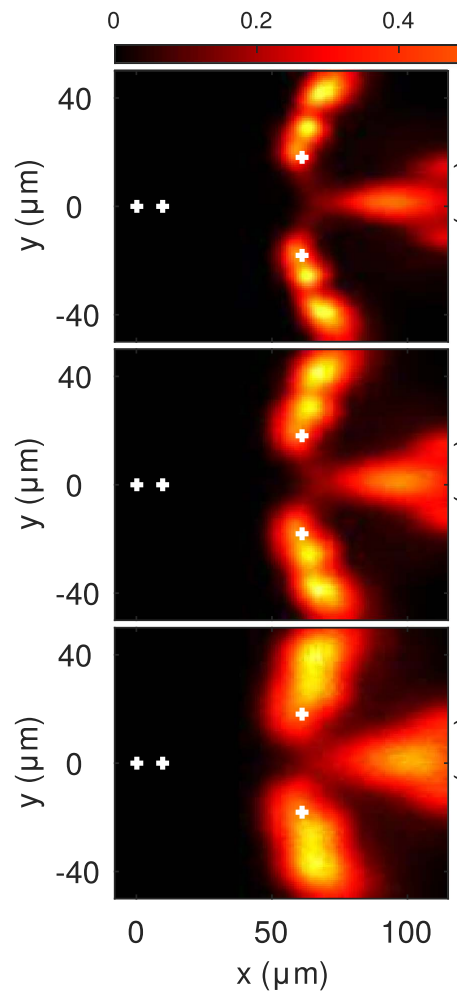

$\begin{array}{lll}0.6 & 0.8\end{array}$
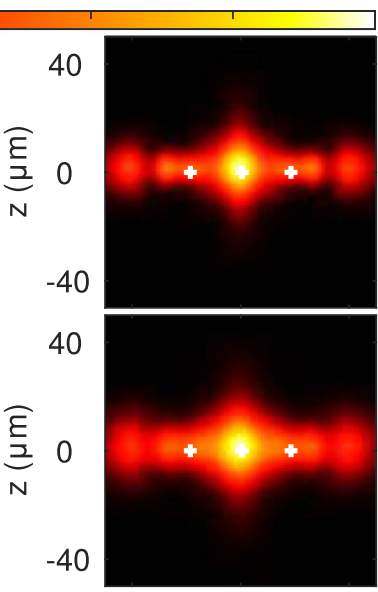

40

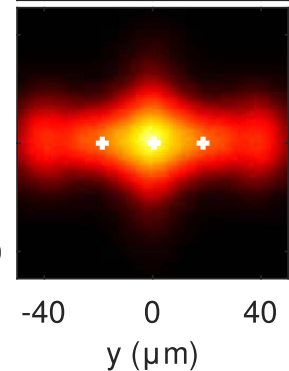

(b)

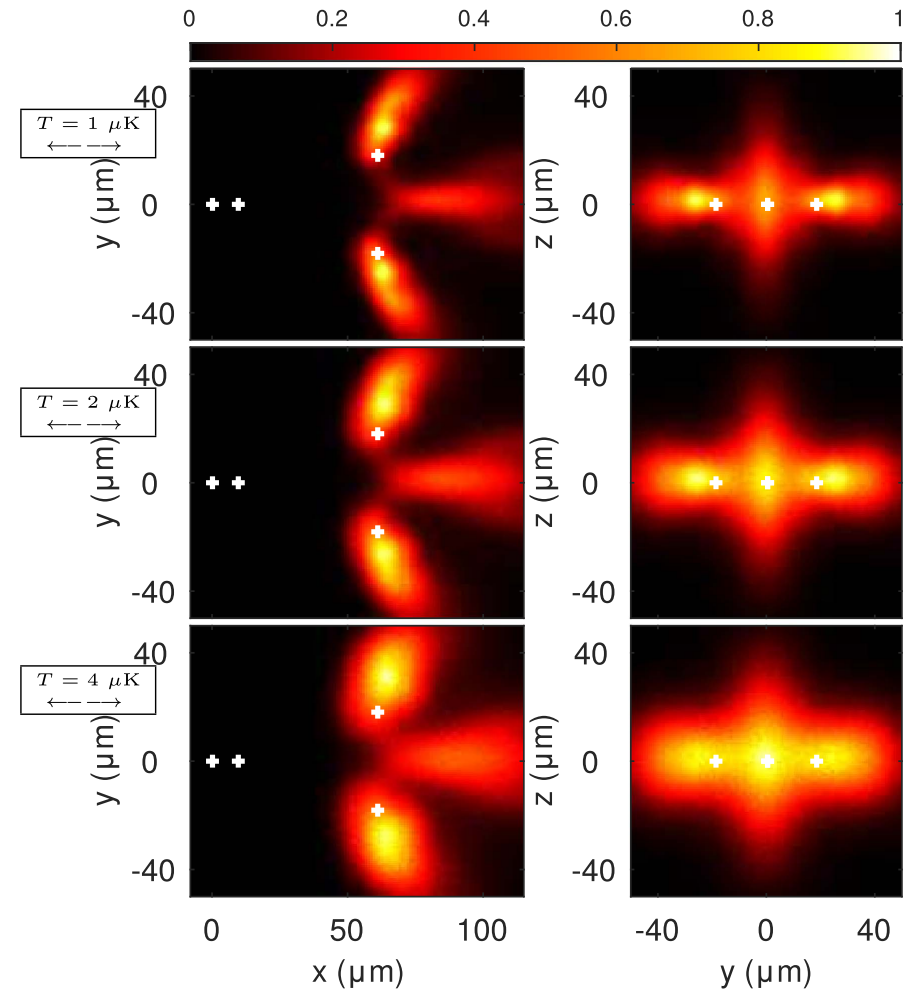

Figure 6. Atomic density for increasing temperatures (top to bottom) and two different waist sizes of the excitation laser: (a) $\sigma_{0}=0.5 \mu \mathrm{m}$. (b) $\sigma_{0}=1 \mu \mathrm{m}$. Shown are the $x-y$ column density (left column) and $y-z$ column density (right column) in (a) and (b), respectively.

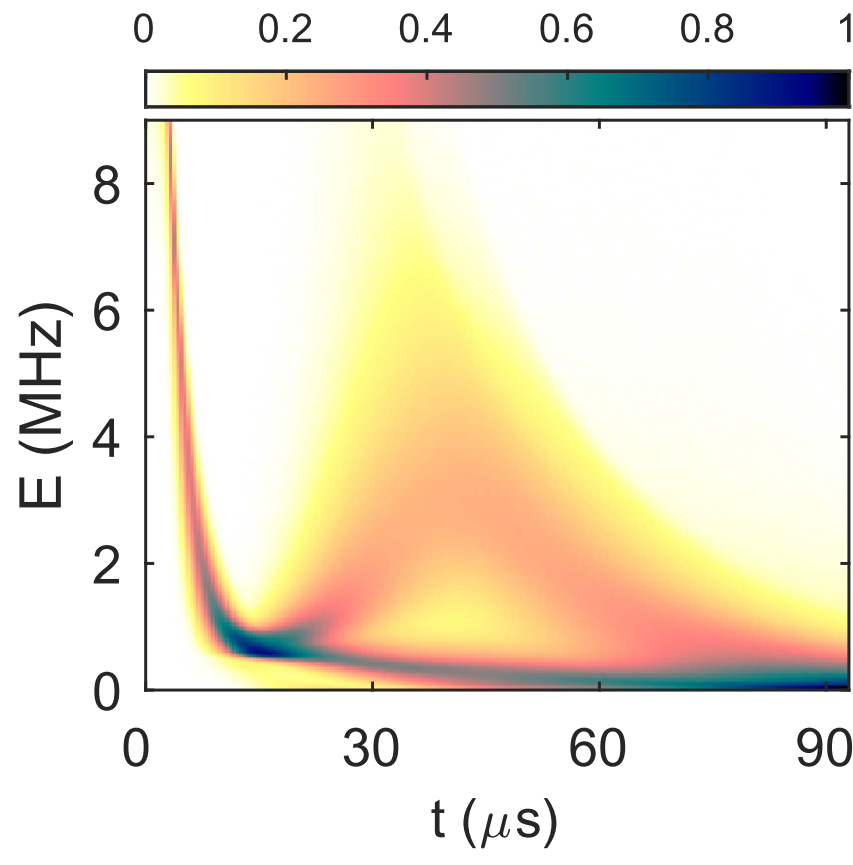

Figure 7. Time-resolved spectrum of potential energy as in figure 5 but for temperature $T=4 \mu \mathrm{K}$ and laser waist size of $\sigma_{0}=1 \mu \mathrm{m}$.

\section{Conclusions and outlook}

In summary, controlled creation of a few Rydberg atoms in a cold gas of ground state atoms will allow to initiate coherent motion of the Rydberg atoms without external confinement as
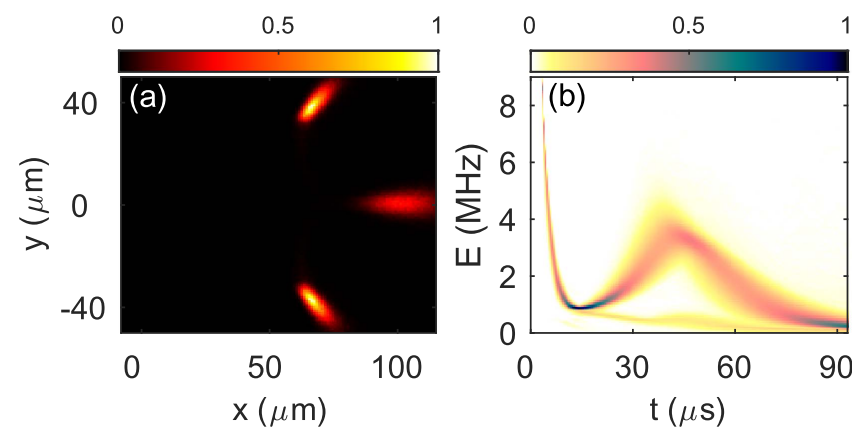

Figure 8. Alternate dynamics for parameters as in section 4 but with microwave polarisation direction $\mathbf{q}=\mathbf{e}_{z}$. (a) Column density in the $x-y$ plane at $t=92.9 \mu \mathrm{s}, n(x, y, t)$. (b) Potential energy density $u(E, t)$, plotted as in figure $5(\mathrm{a})$.

demonstrated here with the unconstrained motion of four Rydberg atoms, forming coupled excitonic BO surfaces. This enables non-adiabatic motional dynamics in assemblies of a few Rydberg excited atoms as an experimental platform for studies of quantum chemical processes inflated to convenient time (microseconds) and spatial (micrometres) scales, with the perspective to shed new light on relevant processes such as ultra-fast vibrational relaxation [55] or reaction control schemes [56]. Experimental observables are atomic density distributions or exciton spectra.

The effects explored will be most prominent with light Alkali species, such as Li discussed here, but also the more common $\mathrm{Rb}$ can be used. Here, a slightly smaller setup would 
sufficiently accelerate the motion to fit our scenario into the $\mathrm{Rb}$ system life-time. $\mathrm{Rb}$ would, however, pose a greater challenge for the theoretical modelling, making the inclusion of spin-orbit coupling necessary [44, 45].

Since the CI in the arrangement discussed here is due to symmetry, it will occur regardless of the precise form of the dipole-dipole interactions. For instance, using a $d$-excitation (angular momentum $l=2$ ) in a $p$-Rydberg aggregate should produce qualitatively similar results. For an $s$-excitation in a $p$-Rydberg aggregate, the results should even agree quantitatively, since a swap of $p$ - and $s$-states leaves the dipoledipole interaction unchanged. However, a symmetric splitting of population onto two surfaces always requires fine-tuning of geometric parameters and would thus depend on details of interaction potentials.

Beyond the selective Rydberg atom activation discussed here, illuminating an entire 3D gas with a single Rydberg excitation laser, followed by microwave transitions to the $p$-state, should also quickly result in non-adiabatic effects. They would arise through the abundant number of CIs in random 3D Rydberg assemblies [20].

\section{Acknowledgments}

We acknowledge helpful discussions with Thomas Pohl.

\section{Appendix A. Excitons and BO surfaces}

The eigenstates of the electronic Hamiltonian equation (3) for a fixed arrangement $\mathbf{R}$ of atoms are termed Frenkel excitons [57]. We label them $\left|\varphi_{k}(\mathbf{R})\right\rangle$ and the corresponding eigenenergies $U_{k}(\mathbf{R})$, defined through

$$
\hat{H}_{\mathrm{el}}(\mathbf{R})\left|\varphi_{k}(\mathbf{R})\right\rangle=U_{k}(\mathbf{R})\left|\varphi_{k}(\mathbf{R})\right\rangle .
$$

The $U_{k}$ are also referred to as BO surfaces. Since our electronic basis $\mathcal{B}$ has $3 N$ elements for $N$ atoms, the operator $\hat{H}_{\mathrm{el}}(\mathbf{R})$ is represented by the $3 N \times 3 N$ matrix

$$
\mathbf{H}_{\mathrm{el}}(\mathbf{R})=\left(\begin{array}{ccc}
h_{1,-1 ; 1,-1}(\mathbf{R}) & \ldots & h_{1,-1 ; N, 1}(\mathbf{R}) \\
\vdots & \ldots & \vdots \\
h_{N, 1 ; 1,-1}(\mathbf{R}) & \ldots & h_{N, 1 ; N, 1}(\mathbf{R})
\end{array}\right)
$$

with $h_{\alpha, m ; \beta, m^{\prime}}(\mathbf{R}):=\left\langle\pi_{\alpha}, m\left|\hat{H}_{\mathrm{el}}(\mathbf{R})\right| \pi_{\beta}, m^{\prime}\right\rangle$. The full electronic wavefunction can be expanded in the eigenstates

$$
\left|\psi_{\mathrm{el}}\right\rangle=\sum_{k=1}^{3 N} \tilde{c}_{k}\left|\varphi_{k}(\mathbf{R})\right\rangle
$$

where the $\tilde{c}_{n}$ are called the adiabatic expansion coefficients. Since for each atom arrangement $\mathbf{R}$ the adiabatic eigenstates and the diabatic basis are linked via a unitary transformation, we can expand the electronic wavefunction also diabatically, i.e.

$$
\left|\psi_{\mathrm{el}}\right\rangle=\sum_{\alpha=1}^{N} \sum_{m=-1}^{1} c_{\alpha, m}\left|\pi_{\alpha}, m\right\rangle,
$$

with $c_{\alpha, m}$ the diabatic coefficients.

\section{Appendix B. Propagation}

The full dynamics is governed by the Hamiltonian

$$
\hat{H}(\mathbf{R})=-\sum_{n=1}^{N} \frac{\nabla_{\mathbf{R}_{n}}^{2}}{2 M}+\hat{H}_{\mathrm{el}}(\mathbf{R}) .
$$

For more than a couple of atoms, solving the time-dependent Schrödinger equation following from equation (B.1) is not feasible in a reasonable time. However a quantum-classical propagation method, Tully's fewest switching algorithm [5860], gives results in good agreement with the full propagation of the Schrödinger equation where possible [17, 19, 23, 27]. In Tully's fewest switching algorithm, the positions $\mathbf{R}$ of the atoms are treated classically according to Newton's equation

$$
M \ddot{\mathbf{R}}=-\nabla_{\mathbf{R}} U_{s}(\mathbf{R}) .
$$

Here, the atoms are subject to a mechanical potential that corresponds to a single eigenenergy $U_{s}$ of the electronic Hamiltonian. The index $s$ will undergo stochastic dynamics described below, for which one needs to calculate a large number of trajectories (solutions) of equation (B.2). The electronic state of the Rydberg aggregate is described quantum mechanically through the electronic Schrödinger equation

$$
\mathrm{i} \hbar \frac{\partial}{\partial t}\left|\psi_{\mathrm{el}}(t)\right\rangle=\hat{H}_{\mathrm{el}}(\mathbf{R}(t))\left|\psi_{\mathrm{el}}(t)\right\rangle
$$

where $\mathbf{R}(t)$, the solution of (B.2), enters as a parameter with $\mathbf{H}_{\mathrm{el}}$ given in equation (A.2). We solve (B.3) by expanding $\left|\psi_{\mathrm{el}}(t)\right\rangle$ in the diabatic basis $\mathcal{B}$, arriving at

$$
\mathrm{i} \hbar \dot{\mathbf{c}}(t)=\mathbf{H}_{\mathrm{el}}(\mathbf{R}(t)) \mathbf{c}(t) .
$$

To retain further quantum properties two features are added. Firstly, the atoms are randomly placed according to the Wigner distribution of the initial nuclear wavefunction and also receive a corresponding random initial velocity. In the end of the simulation, all observables have to be averaged over all realisations. Secondly, non-adiabatic processes are added as follows: The probability for a transition from surface $l$ to surface $k$, is proportional to the non-adiabatic coupling vector

$$
\mathbf{d}_{k l}(\mathbf{R})=\left\langle\varphi_{k}(\mathbf{R})\left|\nabla_{\mathbf{R}}\right| \varphi_{l}(\mathbf{R})\right\rangle .
$$

This coupling is realised in Tully's algorithm by allowing for jumps of the index $s$, from an energy surface $s=l$ to an energy surface $s=k$, during the propagation. The sequence of propagation is as follows:

(i) The initial positions of the atoms are randomly determined in accordance with the probability distribution $\rho(\mathbf{R}, t=0)$ given in (1). The initial velocities are also normally distributed $\dot{\mathbf{R}} \sim \mathcal{N}\left(0, \sigma_{v}^{2}\right)$, with the variance of velocity, $\sigma_{v}^{2}=k_{\mathrm{B}} T / M$, set in agreement with an ideal, classical gas at temperature $T$.

(ii) The electronic Hamiltonian is diagonalized and we pick the electronic state with index $k$ randomly according to the probability $\left|\left\langle\varphi_{\text {ini }} \mid \varphi_{k}\left(\mathbf{R}_{0}\right)\right\rangle\right|^{2}$, where $\left|\varphi_{\text {ini }}\right\rangle$ is the electronic initial state defined in section 2.2. 
(iii) The atomic positions are propagated one time step with equation (B.2), while states are propagated with equation (B.4).

(iv) We determine whether the surface index $s$ undergoes a stochastic jump according to equation (B.5) (see [16] for the precise prescription).

(v) The new positions lead to new eigenstates and -energies, thus we repeat from (ii).

\section{Appendix C. Microwave excitation to the initial electronic state}

With a microwave $\mathcal{E}_{0}(t)$ that is linearly polarised in the $\mathbf{q}$-direction, the aggregate can be excited from the state $|S\rangle$ to an exciton. The Hamiltonian of the microwave-atom coupling can approximately be written as

$$
\hat{H}_{\mathrm{mw}}(t)=\mathcal{E}_{0}(t) \sum_{\alpha=1}^{N} \hat{d}_{0}^{(\alpha)},
$$

where $\hat{d}_{0}^{(\alpha)}$ is the dipole operator of the $\alpha$ th atom projected onto the polarisation direction of the microwave. The relative probability to excite from state $|S\rangle$ with all Rydberg electrons in (the same) $s$-state into a specific exciton state, $\left|\varphi_{k}\right\rangle$, $k \neq 0$ with energy $U_{k}$, can be calculated via $^{5}$

$$
\mathbb{P}\left(|S\rangle,\left|\varphi_{k}\right\rangle\right)=\frac{\left|T\left(|S\rangle,\left|\varphi_{k}\right\rangle\right)\right|^{2} X\left(U_{k}\right)}{\sum_{l=1}^{3 N}\left|T\left(|S\rangle,\left|\varphi_{l}\right\rangle\right)\right|^{2} X\left(U_{l}\right)},
$$

with $T(|S\rangle,|\varphi\rangle):=\left\langle S\left|\hat{H}_{\mathrm{mw}}(t)\right| \varphi\right\rangle$ the transition matrix element from $|S\rangle \rightarrow|\varphi\rangle$ due to the microwave and $X(E)$ the power spectral density of the microwave at energy $E$. Typically we can assume $X(E)$ to be a Gaussian, centred at the microwave frequency (energy) $\hbar \omega_{\mathrm{mw}}$. The transition matrix element is given by

$$
T(|S\rangle,|\varphi\rangle)=\frac{\mathcal{E}_{0}(t) \mathfrak{d}}{\sqrt{3}} \sum_{\alpha=1}^{N} c_{\alpha, 0},
$$

with $c_{\alpha, 0}=\left\langle\pi_{\alpha}, 0 \mid \varphi\right\rangle$ the diabatic expansion coefficients of the exciton. The initial atomic configuration is chosen, such that there are excitons localised on the $x$-dimer which provide repulsive interactions. According to (C.3), the microwave can only excite to excitons with excitation oriented along the microwave polarisation direction. Only for $\mathbf{q} \in\left\{\mathbf{e}_{y}, \mathbf{e}_{z}\right\}$ there is a single repulsive exciton, localised on the $x$-dimer, with even electronic symmetry

$$
|\varphi\rangle \approx\left(\left|\pi_{1}, 0\right\rangle+\left|\pi_{2}, 0\right\rangle\right) / \sqrt{2}
$$

The latter is required for the transition according to (C.3) to be allowed such that we can initially excite to this state, $\left|\psi_{\mathrm{el}}(t=0)\right\rangle=|\varphi\rangle$, by choosing a microwave frequency of $\omega_{\mathrm{mw}}=22.27 \mathrm{MHz}$.

${ }^{5}$ We use $U_{k} \ll \hbar \omega_{\mathrm{mw}}$.

\section{Appendix D. Formulas for atomic density and spatial distribution of the p-excitation}

In the following we specify several quantities used in the main text. The atomic density is defined as

$$
n(\mathbf{r}, t):=\left.\frac{1}{N} \sum_{j=1}^{N} \int \mathrm{d}^{N-1} \mathbf{R}_{\{j\}} \rho(\mathbf{R}, t)\right|_{\mathbf{R}_{j}=\mathbf{r}},
$$

where

$$
\begin{aligned}
\rho(\mathbf{R}, t) & :=\left\langle\mathbf{R}\left|\varrho^{\text {nuc }}(t)\right| \mathbf{R}\right\rangle \\
& =\sum_{k, m}\left\langle\mathbf{R} ; \pi_{k}, m|\hat{\varrho}(t)| \mathbf{R} ; \pi_{k}, m\right\rangle
\end{aligned}
$$

is the probability distribution of the atomic positions $\mathbf{R}$ at time $t$. Note that we used the abbreviation $\left|\mathbf{R} ; \pi_{k}, m\right\rangle:=$ $|\mathbf{R}\rangle \otimes\left|\pi_{k}, m\right\rangle$. The integration $\int \mathrm{d}^{N-1} \mathbf{R}_{\{j\}}$ is over all but the coordinates of the $j$ th atom. The corresponding column densities are obtained by integrating out the direction which shall be removed. For instance, the atomic $x-y$ column density is given by $\bar{n}(x, y, t)=\int \mathrm{d} z n(\mathbf{r}=(x, y, z), t)$.

The $m$-level segregated spatial distributions of the $p$ excitation are defined as

$$
\begin{aligned}
\rho_{\mathrm{exc}, m}(\mathbf{r}, t):= & \frac{1}{N} \sum_{j=1}^{N} \int \mathrm{d}^{N-1} \mathbf{R}_{\{j\}} \\
& \times\left.\left\langle\mathbf{R} ; \pi_{j}, m|\hat{\varrho}(t)| \mathbf{R} ; \pi_{j}, m\right\rangle\right|_{\mathbf{R}_{j}=\mathbf{r}}
\end{aligned}
$$

and the corresponding column densities are obtained in the same way as for the atomic density. In the context of Tully's surface hopping, as described in appendix B, densities are calculated as follows: for each trajectory the relevant quantity is binned in a predefined array and subsequently normalised by dividing through the number of trajectories. For the atomic density, the relevant quantity is the atomic configuration of the aggregate, whereas for the spatial excitation density, before binning, the position of each atom is weighted with the probability that the respective atom carries excitation. For more details of the procedure, see the supplemental information of [27].

\section{Appendix E. Inelastic interactions with the background gas}

For the scenario proposed here, Rydberg excited atoms with $\nu=80$ in $l=0,1$ states move through a background gas of ground state atoms with density $\rho=1 \times 10^{12} \mathrm{~cm}^{-3}$ at a maximal velocity of about $v_{\text {ini }} \sim \sqrt{U_{\text {ini }}\left(R_{0}\right) / 2} \approx 0.85 \mathrm{~m} \mathrm{~s}^{-1}$. We can deduce a maximal cross-section for ionising collisions between Rydberg atoms and ground state atoms of $\sigma(\nu)=610 \mathrm{~nm}^{2}$ at $\nu=60$ from experiment [34]. Assuming scaling with the size of the Rydberg orbit [52], we extrapolate this value to our $\nu=80$, thus $\sigma(80)=\sigma(60)(80 / 60)^{2} 10^{3} ; \mathrm{nm}^{2}$.

The total decay rate of our four atom system is then $\Gamma_{\text {tot }}=2 \Gamma_{\text {coll }}+4 \Gamma_{0}$, with spontaneous decay rate $\Gamma_{0}$ and collisional decay rate $\Gamma_{\text {coll }}$ for single atoms. We have assumed that only two atoms ever move with the fastest velocity. Using $\Gamma_{\text {coll }}=\rho v_{\text {ini }} \sigma(80)$, we finally arrive at a total life-time $\tau=1 / \Gamma_{\text {tot }}=530 \mu \mathrm{s}$ as quoted in the main text. 


\section{References}

[1] Gallagher T F 1994 Rydberg Atoms (Cambridge: Cambridge University Press)

[2] Browaeys A, Barredo D and Lahaye T 2016 J. Phys. B: Atom. Mol. Opt. Phys. 49152001

[3] Jaksch D et al 2000 Phys. Rev. Lett. 852208

[4] Lukin M D et al 2001 Phys. Rev. Lett. 87037901

[5] Urban E et al 2009 Nat. Phys. 5110

[6] Gaëtan A et al 2009 Nat. Phys. 5115

[7] Lesanovsky I and Garrahan J P 2013 Phys. Rev. Lett. 111215305

[8] van Bijnen R M W and Pohl T 2015 Phys. Rev. Lett. 114 243002

[9] Glaetzle A W et al 2015 Phys. Rev. Lett. 114173002

[10] Wilk T et al 2010 Phys. Rev. Lett. 104010502

[11] Müller M M et al 2014 Phys. Rev. A 89032334

[12] Mülken O et al 2007 Phys. Rev. Lett. 99090601

[13] Barredo D et al 2015 Phys. Rev. Lett. 114113002

[14] Bettelli S et al 2013 Phys. Rev. A 88043436

[15] Günter G et al 2013 Science 342954

[16] Ates C, Eisfeld A and Rost J M 2008 New J. Phys. 10045030

[17] Wüster S, Ates C, Eisfeld A and Rost J M 2010 Phys. Rev. Lett. 105053004

[18] Leonhardt K, Wüster S and Rost J M 2016 Phys. Rev. A 93 022708

[19] Möbius S et al 2011 J. Phys. B: At. Mol. Opt. Phys. 44184011

[20] Wüster S, Eisfeld A and Rost J M 2011 Phys. Rev. Lett. 106 153002

[21] Wüster S, Ates C, Eisfeld A and Rost J M 2011 New J. Phys. 13073044

[22] Zoubi H, Eisfeld A and Wüster S 2014 Phys. Rev. A 89053426

[23] Möbius S et al 2013 Phys. Rev. A 88012716

[24] Wüster S et al 2013 Phys. Rev. A 88063644

[25] Genkin M et al 2014 J. Phys. B: At. Mol. Opt. Phys. 47095003

[26] Möbius S et al 2013 Phys. Rev. A 87051602

[27] Leonhardt K, Wüster S and Rost J M 2014 Phys. Rev. Lett. 113 223001

[28] Domcke W, Yarkony D R and Köppel H 2004 Conical Intersections (Singapore: World Scientific)

[29] White A J, Peskin U and Galperin M 2013 Phys. Rev. B 88 205424

[30] Olmos B, Li W, Hofferberth S and Lesanovsky I 2011 Phys. Rev. A 84 041607(R)

[31] Günter G et al 2012 Phys. Rev. Lett. 108013002
[32] Schönleber D W et al 2015 Phys. Rev. Lett. 114123005

[33] Schempp H et al 2015 Phys. Rev. Lett. 115093002

[34] Celistrino Teixeira R et al 2015 Phys. Rev. Lett. 115 013001

[35] Thaicharoen N, Schwarzkopf A and Raithel G 2015 Phys. Rev. A 92 040701(R)

[36] Thaicharoen N, Gonçalves L F and Raithel G 2016 Phys. Rev. Lett. 116213002

[37] Fioretti A et al 1999 Phys. Rev. Lett. 821839

[38] Li W, Tanner P J and Gallagher T F 2005 Phys. Rev. Lett. 94 173001

[39] Mudrich M et al 2005 Phys. Rev. Lett. 95233002

[40] Marcassa L G, de Oliveira A L, Weidemüller M and Bagnato V S 2005 Phys. Rev. A 71054701

[41] Nascimento V A et al 2006 Phys. Rev. A 73034703

[42] Amthor T et al 2007 Phys. Rev. Lett. 98023004

[43] Amthor T, Reetz-Lamour M, Giese C and Weidemüller M 2007 Phys. Rev. A 76054702

[44] Park H et al 2011 Phys. Rev. A 84022704

[45] Park H, Shuman E S and Gallagher T F 2011 Phys. Rev. A 84 052708

[46] Lühmann D-S, Weitenberg C and Sengstock K 2015 Phys. Rev. X, 5031016

[47] Goy P, Liang J and Haroche S 1986 Phys. Rev. A 342889

[48] Robicheaux F, Hernandez J V, Topcu T and Noordam L D 2004 Phys. Rev. A 70042703

[49] Nogrette F et al 2014 Phys. Rev. X, 4021034

[50] Greene C H, Dickinson A S and Sadeghpour H R 2000 Phys. Rev. Lett. 852458

[51] Balewski J B et al 2013 Nature 502664

[52] Niederprüm T et al 2015 Phys. Rev. Lett. 115013003

[53] Grünzweig T, Hilliard A, McGovern M and Andersen M F 2010 Nat. Phys. 6951

[54] Kaufman A M, Lester B J and Regal C A 2012 Phys. Rev. X 2 041014

[55] Perun S, Sobolewski A L and Domcke W 2005 Chem. Phys. 313107

[56] Epshtein M, Yifrach Y, Portnov A and Bar I 2016 J. Phys. Chem. Lett. 71717

[57] Frenkel J 1931 Phys. Rev. 3717

[58] Tully J C and Preston R K 1971 J. Chem. Phys. 55562

[59] Hammes-Schiffer S and Tully J C 1994 J. Chem. Phys. 1014657

[60] Barbatti M 2011 Wiley Interdiscipl. Rev. Comput. Mol. Sci. 1620 